University of Wollongong

Research Online

Faculty of Engineering - Papers (Archive)

Faculty of Engineering and Information

Sciences

$1-1-2007$

\title{
Spin-orbit interaction enhanced polaron effect in two-dimensional semiconductors
}

Zhou Li

Peking University, zli@pku.edu.cn

Zhongshui Ma

Peking University, zma@uow.edu.au

Anthony Wright

University of Wollongong, arw75@uow.edu.au

Chao Zhang

University of Wollongong, czhang@uow.edu.au

Follow this and additional works at: https://ro.uow.edu.au/engpapers

Part of the Engineering Commons

https://ro.uow.edu.au/engpapers/1766

\section{Recommended Citation}

Li, Zhou; Ma, Zhongshui; Wright, Anthony; and Zhang, Chao: Spin-orbit interaction enhanced polaron effect in two-dimensional semiconductors 2007, 112103-1-112103-3.

https://ro.uow.edu.au/engpapers/1766

Research Online is the open access institutional repository for the University of Wollongong. For further information contact the UOW Library: research-pubs@uow.edu.au 


\title{
Spin-orbit interaction enhanced polaron effect in two-dimensional semiconductors
}

\author{
Zhou Li and Zhongshui Ma \\ School of Physics, Peking University, Beijing 100871, China
}

\begin{abstract}
A. R. Wright and Chao Zhang ${ }^{\text {a) }}$
School of Engineering Physics, University of Wollongong, New South Wales 2522, Australia
\end{abstract}

(Received 9 January 2007; accepted 8 February 2007; published online 13 March 2007)

\begin{abstract}
It is shown that in two-dimensional semiconductors, the electron-phonon interaction and polaron mass correction are both significantly enhanced by the Rashba spin-orbit coupling. The mass correction is positive for the upper Rashba branch and negative for the lower Rashba branch. Both Rashba branches have the same polaron binding energy, which is higher than that for systems in the absence of spin-orbit interaction. To the leading order, the correction to the binding energy is proportional to the square of the spin-orbit coupling strength. (C) 2007 American Institute of Physics. [DOI: $10.1063 / 1.2713346$ ]
\end{abstract}

The recent advancement in spin electronics (spintronics) and spintronic materials ${ }^{1}$ has provided opportunities in developing optoelectronic devices whose optical properties can be tuned by the intrinsic spin-orbit interaction. Spintronic material systems have been realized and many advanced electronic devices have been proposed recently, such as spin transistors, ${ }^{2}$ spin waveguides, ${ }^{3}$ spin filters, ${ }^{4}$ etc. In narrowgap semiconductor nanostructures, such as InAs and $\mathrm{In}_{1-x} \mathrm{Ga}_{x}$ As quantum wells, the inversion asymmetry of the confining potential due to the presence of the heterojunction ${ }^{5}$ results in the spin splitting (or spontaneous spin splitting) of the carriers in the absence of any applied magnetic field. Such an effect is similar to that under an inhomogeneous surface electric field. Therefore the inhomogeneous heterostructure confining potential is an electrical equivalence to the Rashba spin splitting or Rashba effect. ${ }^{6}$ This confining potential can be further tuned with an applied gate voltage. Phenomena related to Rashba effects in, e.g., InAs- and $\mathrm{In}_{1-x} \mathrm{Ga}_{x} \mathrm{As}$-based two-dimensional electron gas (2DEG) systems have been observed. ${ }^{7,8}$ The value of the Rashba parameter in these semiconductor systems can be as high as 4 $\times 10^{-11} \mathrm{eV} \mathrm{m}$. The experimental results ${ }^{7,8}$ have shown that in InAs- and $\operatorname{In}_{1-x} \mathrm{Ga}_{x} \mathrm{As}$-based $2 \mathrm{DEG}$ systems, the Rashba effect is responsible for the spontaneous spin splitting. Other contributions such as the Dresselhaus term can also be significant in systems with wide gaps, because they come mainly from the bulk-inversion asymmetry of the material. ${ }^{9}$ We have shown recently that the spin-orbit interaction (SOI) can lead to a finite transverse electrical current in the absence of any magnetic field. ${ }^{10}$ The SOI can also be used to produce spin polarized current in a magnetic superlattice. ${ }^{11}$ Under the SOI, the optical spectral weight among various collective excitations $^{12}$ can be tuned.

Electron-phonon interaction plays an important role in a number of physical phenomena such as superconductivity, magnetophonon anomalies, ${ }^{13}$ polaronic effect, localization, hot carrier transport, ${ }^{14}$ and hypersound generation. ${ }^{15}$ Electron longitudinal-optical (LO)-phonon scattering also plays a significant role in inelastic light scattering in semiconductor quantum wells because of the coupling between the LO

${ }^{a)}$ Electronic mail: czhang@uow.edu.au phonons and the intersubband plasmons. In this letter we investigate the electron-LO-phonon interaction and the polaron effect in two-dimensional semiconductors with a Rashba SOI. Our results show that the polaron effect can be significantly enhanced by the SOI.

We consider a two-dimensional electronic system in the $x-y$ plane in narrow-gap semiconductor nanostructures (e.g., InGaAs/InAlAs quantum wells). The Hamiltonian of a free electron is given as

$$
H_{0}=\frac{1}{2 m^{*}}\left(p_{x}^{2}+p_{y}^{2}\right)+\frac{\alpha}{\hbar}\left(\sigma_{y} p_{x}-\sigma_{x} p_{y}\right),
$$

where $m^{*}$ is the electron effective mass in the absence of electron-phonon interaction and SOI, $\alpha$ is the Rashba SOI parameter, $\sigma_{x}$ and $\sigma_{y}$ are Pauli spin matrices, and $p_{x}$ and $p_{y}$ are the electron momentum operators. $p_{x}$ is taken to be along the [100] direction and $p_{y}$ along the [010] direction; the wave function can be written in the form of $\psi(x, y)=u_{\mathbf{k}}(x, y) \xi_{k, \lambda}$, where $u_{\mathbf{k}}(x, y)$ is given by $u_{\mathbf{k}}(x, y)=\exp \left(i k_{x} x+i k_{y} y\right)$ because of translation invariance. The eigenvalue is

$$
\varepsilon_{\lambda}^{(0)}=\frac{\hbar^{2} k^{2}}{2 m^{*}}+\lambda \alpha k,
$$

with $k=\sqrt{k_{x}^{2}+k_{y}^{2}}$ and $\lambda= \pm 1$. The corresponding eigenfunctions of spinors $\xi_{k, \lambda}$ are found to be

$$
\xi_{k, \lambda}=\frac{1}{\sqrt{2}}\left(\begin{array}{c}
1 \\
-\lambda\left(k_{y}-i k_{x}\right) / k
\end{array}\right) .
$$

Using these eigenfunctions the electron quantum field operators are constructed, $\hat{\Psi}(x, y)=\Sigma_{k, \lambda} \hat{a}_{\mathbf{k} \lambda} u_{\mathbf{k}}(x, y) \xi_{\mathbf{k} \lambda}$ and $\hat{\Psi}^{\dagger}(x, y)=\sum_{k, \lambda} \hat{a}_{\mathbf{k} \lambda}^{\dagger} u_{\mathbf{k}}^{*}(x, y) \xi_{\mathbf{k} \lambda}^{*}$, where $\hat{a}_{\mathbf{k} \lambda}\left(\hat{a}_{\mathbf{k} \lambda}^{\dagger}\right)$ is the creation (annihilation) operator for an electron with wave number $\mathbf{k}$ and spin state $\lambda$. The electron-LO-phonon interaction in semiconductors is modeled by the Fröhlich coupling,

$$
H_{e-\mathrm{ph}}=\sum_{k, \lambda} M(q) \hat{a}_{\mathbf{k}+\mathbf{q} \lambda}^{\dagger} \hat{a}_{\mathbf{k} \lambda}\left(\hat{b}_{\mathbf{q}}+\hat{b}_{-\mathbf{q}}^{\dagger}\right),
$$

where $\hat{b}_{\mathbf{q}}^{\dagger}$ and $\hat{b}_{\mathbf{q}}$ are the creation and annihilation operators for the LO phonons. The coupling strength is given as $M(q)=\sqrt{2 \pi e^{2} \hbar \omega_{\mathrm{LO}} / q\left(1 / \epsilon_{\infty}-1 / \epsilon_{s}\right)}$. Here $\epsilon_{\infty}$ and $\epsilon_{s}$ are the 
high frequency and static dielectric constants of the semiconductor. The electron energy including the self-energy correction due to the electron-LO-phonon interaction is described by the Rayleigh-Schrödinger perturbation theory: ${ }^{16}$

$$
\varepsilon_{\mathbf{k}, \lambda}=\varepsilon_{\mathbf{k}, \lambda}^{0}-\sum_{\mathbf{q}, \lambda^{\prime}} \frac{|M(q)|^{2}\left|g^{\lambda, \lambda^{\prime}}(\mathbf{k}, \mathbf{k}+\mathbf{q})\right|^{2}}{\varepsilon_{\mathbf{k}+\mathbf{q}, \lambda^{\prime}}^{0}-\varepsilon_{\mathbf{k}, \lambda}^{0}+\hbar \omega_{\mathrm{LO}}},
$$

where $\omega_{\mathrm{LO}}$ is the frequency of the LO phonons and the structure factor is given by

$$
\left|g^{\lambda, \lambda^{\prime}}(\mathbf{k}, \mathbf{k}+\mathbf{q})\right|^{2}=\frac{1}{2}\left[1+\lambda \lambda^{\prime} \frac{k+q \cos \varphi}{\sqrt{k^{2}+q^{2}+2 k q \cos \varphi}}\right] .
$$

The $\varphi$ is the angle between the $\mathbf{k}$ vector and the $\mathbf{x}$ axis.

After introducing the following dimensionless parameters, we have $u=k / k_{F}(0), \quad z=q / k_{F}(0)$, $\Omega_{\mathrm{LO}}=2 m_{0} \hbar \omega_{\mathrm{LO}} / \hbar^{2} k_{F}^{2}(0), \quad k_{\alpha}=2 m_{0} \alpha / \hbar^{2}, \quad \alpha_{s}$ $=\left(e^{2} / \hbar\right) \sqrt{m_{0} / 2 \hbar \omega_{\mathrm{LO}}}\left(1 / \epsilon_{\infty}-1 / \epsilon_{s}\right)$, and $y=k_{\alpha} / k_{F}(0)$. The electron energy is then written as

$$
\varepsilon_{\mathbf{u}, \lambda}=\frac{\hbar^{2} k_{F}^{2}(0)}{2 m_{0}}\left[u^{2}+\lambda \frac{k_{\alpha}}{k_{F}(0)} u+\delta \varepsilon\right],
$$

where $\delta \varepsilon$ is the electron self-energy correction due to the electron-LO-phonon interaction, which is found as $\delta \varepsilon=$ $-\left(\Omega_{\mathrm{LO}}^{3 / 2} \alpha_{S} / 4 \pi\right) \Sigma^{\lambda}(\mathbf{u})$ and $\Sigma^{\lambda}(\mathbf{u})=\Sigma_{\lambda^{\prime}} \int_{0}^{\infty} d z \int_{0}^{2 \pi} d \varphi \mid g^{\lambda, \lambda^{\prime}}(\mathbf{u}, \mathbf{u}$ $+\mathbf{z})\left.\right|^{2} /\left(\Omega_{\mathrm{LO}}+\varepsilon_{\mathbf{u}+\mathbf{z}, \lambda^{\prime}}^{0}-\varepsilon_{\mathbf{u}, \lambda}^{0}\right)$. This result contains only the leading order contribution in Rayleigh-Schrödinger perturbation theory for a single electron in either Rashba branch interacting with the polar lattice. The polaron binding energy is obtained as

$$
E_{0}=-\frac{\pi}{2} \alpha_{s} \hbar \omega_{\mathrm{LO}} \frac{1}{\sqrt{1-y^{2} /\left(4 \Omega_{\mathrm{LO}}\right)}} .
$$

It is found that the polaron binding energy is enhanced in systems with finite SOI as compared to that in systems with zero SOI. The enhancement factor is $\left[\sqrt{1-y^{2} /\left(4 \Omega_{\mathrm{LO}}\right)}\right]^{-1}$. To the leading order of SOI, the energy enhancement $\Delta E \propto \alpha^{2}$. The correction to the free electron energy due to $\mathrm{SOI}$ is $\pm \alpha k$. The splitting of the original parabolic band into two Rashba bands provides an efficient channel for LO-phonon scattering via interlevel transitions. In addition, the linear energy correction makes the intralevel phonon scattering more efficient. This result, if confirmed experimentally, is very interesting. It demonstrates an intrinsic correlation between the electronlattice coupling and the spin-orbit coupling.

The polaron effective mass is given as $1 / m_{i j}$ $=\partial^{2} \varepsilon / \partial k_{i} \partial k_{j}$. For a system with SOI, the effective mass is not constant even near the band minimum. The energy (or $k$ ) dependent effective mass is common to electrons and holes where the band dispersions are nonparabolic. ${ }^{17,18}$ Such a nonparabolic dispersion can be caused by various interactions, e.g., high order electron-phonon interaction. In the present system, it is due to both the electron SOI and the electron-phonon interaction. From Eq. (6), the polaron effective mass is obtained,

$$
\left(\frac{1}{m^{*}}\right)_{x x}=\frac{1}{2 m_{0}} \frac{\partial^{2}}{\partial u_{x}^{2}}\left(u^{2}+\frac{\lambda k_{\alpha} u}{k_{F}(0)}-\frac{\alpha_{s} \Omega_{\mathrm{LO}}^{3 / 2} \Sigma^{\lambda}(\mathbf{u})}{4 \pi}\right) .
$$

In Fig. 1, we show the dependence of the inverse effective mass correction at $k=0$ on the Rashba SOI parameter. The dependence is almost linear. For the present system at

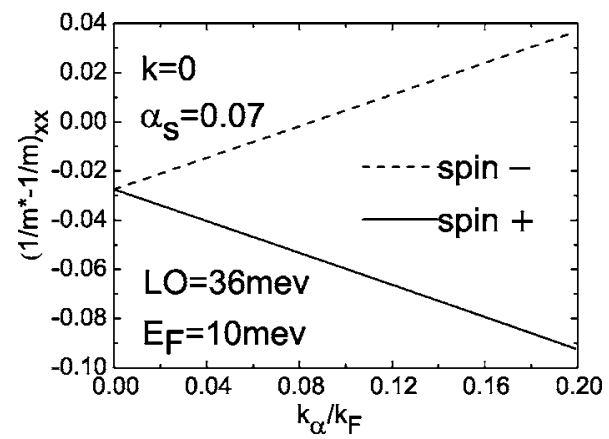

FIG. 1. Polaronic effective mass correction at $k=0$ as a function of the SOI strength.

zero SOI, the electron-LO-phonon interaction results in a polaron effective mass which is slightly heavier than the electron effective mass. The value at zero $\alpha$ in Fig. 1 represents this mass correction. The effective mass of the low Rashba band decreases with the SOI and that of the upper Rashba band increases with the SOI. The average of $1 / m_{x x}^{*}$ (up) and $1 / m_{x x}^{*}$ (down) represents the polaron effective mass in the absence of SOI. The variation of $m_{x x}^{*}$ (up) and $m_{x x}^{*}$ (down) with SOI can be understood as follows. Near $k=0$, electron-phonon interaction generally leads to a reduction of electron energy by an amount around the polaron binding energy. In terms of electron energy dispersion, this reduction in energy is equivalent to an increase in the effective mass. This is the polaron effect in the absence of the SOI. The situation with SOI is quite different. Near $k=0$, the effective mass of the Rashba bands are given as $1 / m_{x x}^{*}$ $=1 / m^{*} \pm \alpha k_{y}^{2} / 2 k^{3}$. Therefore, the electron effective mass of the upper band is reduced by the SOI but remains positive. The lighter effective mass makes the electron-phonon interaction more effective and as a result the polaronic mass correction is enhanced by the SOI (the solid line of Fig. 1). For the lower Rashba band the electron effective mass is heavier than that without SOI and the polaronic mass correction is smaller. Furthermore, in the case of sufficiently strong SOI, the electron effective mass can be negative. In this case lowering the energy further by the electron-phonon interaction actually reduces the effective mass. For the present system, this happens when $k_{\alpha} / k_{F}>0.08$.

Unlike electrons in a parabolic band, the electron and polaron effective masses depend on the magnitude and the direction of wave vector $k$ at small $k$. This $k$ dependence is shown in Figs. 2 and 3. The $\theta$ is the angle between the $\mathbf{k}$ vector and the $\mathrm{x}$ axis. For the upper Rashba band, increasing $k$ will show the interplay of two countereffects. On the one

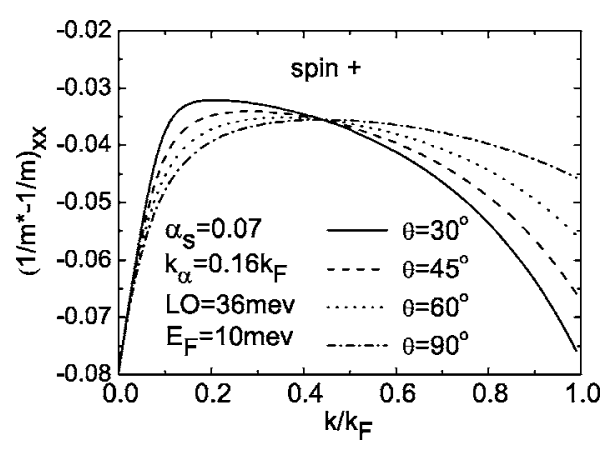

FIG. 2. Wave number dependent polaronic effective mass correction for the upper Rashba band. 


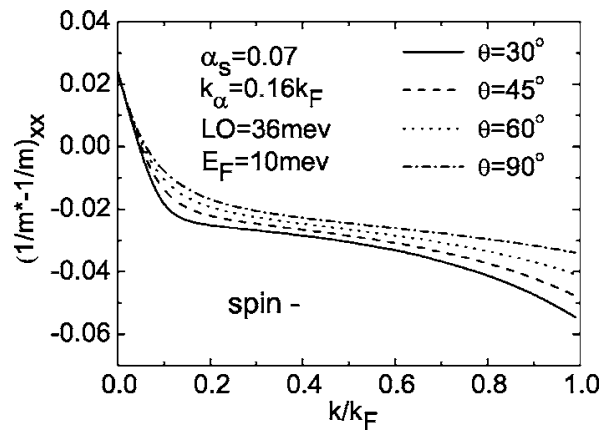

FIG. 3. Wave number dependent polaronic effective mass correction for the lower Rashba band.

hand, the linear- $k$ term in the energy dispersion becomes less important as $k$ increases and thus the polaronic mass correction is reduced. On the other hand, the polaronic mass correction due in a pure parabolic band ${ }^{13}$ increases with $k$. As a result, the polaronic mass correction decreases with $k$ in the small- $k$ regime and increases with $k$ in the large- $k$ region. This behavior is shown in Fig. 2. For the lower Rashba band, the two effects mentioned above are not in a counterplay but rather support each other. As a result, the polaronic mass correction increases with $k$ monotonically. This is shown in Fig. 3.

We suggest to perform far infrared magnetoabsorption measurements ${ }^{19,20}$ on InAs quantum well structures in order to test the enhanced polaron effect reported here. The measurement of effective masses using the far infrared magnetospectroscopy should reveal information about the interaction of an electron with the periodic potential as well as formation about electronic quasiparticles such as polarons. The SOI dependence of the polaron effect can be measured by varying the gate voltage.

In conclusion, we have shown that the SOI leads to quite different polaronic effect in two-dimensional semiconductors. The polaronic binding energy is enhanced by the SOI and the polaronic effective mass varies differently in different Rashba bands.

This work is supported in part by the Australian Research Council, RFDP-China, and NNSFC Grant Nos. 10474002 and 10674004 . The authors thank W. Xu for interesting discussions on the subject.

${ }^{1}$ S. A. Wolf, D. D. Awschalom, R. A. Buhrman, J. M. Daughton, S. von Molnr, M. L. Roukes, A. Y. Chtchelkanova, and D. M. Treger, Science 294, 1488 (2001).

${ }^{2}$ B. Datta and S. Das, Appl. Phys. Lett. 56, 665 (1990).

${ }^{3}$ X. F. Wang, P. Vasilopoulos, and F. M. Peeters, Phys. Rev. B 65, 165217 (2002).

${ }^{4}$ T. Koga, J. Nitta, H. Takayanagi, and S. Datta, Phys. Rev. Lett. 88, 126601 (2002)

${ }^{5}$ Th. Schners, G. Engels, J. Lange, Th. Klocke, M. Hollfelder, and H. Lüth, J. Appl. Phys. 83, 4324 (1998).

${ }^{6}$ E. I. Rashba, Fiz. Tverd. Tela (Leningrad) 2, 1224 (1960) E. I. Rashba [Sov. Phys. Solid State 2, 1109 (1960)]; E. I. Rashba and V. I. Sheka, in Landau Level Spectroscopy, edited by G. Landwehr and E. I. Rashba (North-Holland, Amsterdam, 1991), Vol. 1, p. 131.

${ }^{7}$ Y. Sato, T. Kita, S. Gozu, and S. Yamada, J. Appl. Phys. 89, 8017 (2001); D. Grundler, Phys. Rev. Lett. 84, 6074 (2000).

${ }^{8}$ J. Nitta, T. Akazaki, and H. Takayanagi, Phys. Rev. Lett. 78, 1335 (1997).

${ }^{9}$ J. Schliemann, J. C. Egues, and D. Loss, Phys. Rev. B 67, 085302 (2003).

${ }^{10}$ C. Zhang and Z. S. Ma, Phys. Rev. B 71, 121307 (2005).

${ }^{11}$ Jun-Feng Liu, Huatong Yang, Chao Zhang, and Zhongshui Ma, Phys. Rev. B 73, 155309 (2006).

${ }^{12}$ C. H. Yang, A. Wright, F. Gao, C. Zhang, Z. Zeng, and W. Xu, Appl. Phys. Lett. 88, 223102 (2006).

${ }^{13}$ S. Das Sarma and A. Madhukar, Phys. Rev. B 22, 2823 (1980).

${ }^{14}$ W. Xu and C. Zhang, Phys. Rev. B 55, 5259 (1997).

${ }^{15}$ W. Xu and C. Zhang, Appl. Phys. Lett. 68, 823 (1996).

${ }^{16}$ G. D. Mahan, Many-Particle Physics, 3rd ed. (Kluwer Academic, Dordrecht/Plenum, New York, 2000), p. 438.

${ }^{17}$ H. R. Glyde, B. Fk, N. H. van Dijk, H. Godfrin, France K. Guckelsberger, and R. Scherm, Phys. Rev. B 61, 1421 (2000).

${ }^{18}$ T. K. Thirumalaisamy and D. Chandramohan, Phys. Rev. B 44, 6158 (1991).

${ }^{19}$ A. J. L. Poulter, J. Zeman, D. K. Maude, M. Potemski, G. Martinez, A. Riedel, R. Hey, and K. J. Friedland, Phys. Rev. Lett. 86, 336 (2001).

${ }^{20}$ S. Hameau, Y. Guldner, O. Verzelen, R. Ferreira, G. Bastard, J. Zeman, A. Lematre, and J. M. Grard, Phys. Rev. Lett. 83, 4152 (1999). 